Check for updates

Cite this: Phys. Chem. Chem. Phys., 2018, 20, 22980

Received 15th May 2018 Accepted 10th August 2018

DOI: $10.1039 / c 8 c p 03093 f$

rsc.li/pccp

\section{Transport properties and intermolecular interactions in binary mixtures based on the protic ionic liquid ethylimidazolium triflate and ethylene glycol $\uparrow$}

\author{
Negin Yaghini, (D) Iqbaal Abdurrokhman, Mohammad Hasani and \\ Anna Martinelli (D) *
}

\begin{abstract}
The binary mixture based on the protic ionic liquid (PIL) ethylimidazolium triflate $\left(\mathrm{C}_{2} \mathrm{HImTfO}\right)$ and the diol compound ethylene glycol (EG) has been investigated in the whole composition range from pure PIL to pure EG. At $30{ }^{\circ} \mathrm{C}$ the addition of EG increases both the ionic conductivity and the self-diffusivity of the ions. These quantities, however, change at different rates suggesting that the ionicity of the system is composition dependent. This behaviour is explained by means of new intermolecular forces established when a second compound like EG is introduced into the ionic network. More specifically, a complex $\mathrm{H}$-bonded network is formed that involves the $-\mathrm{NH}$ group of the cation, the $-\mathrm{OH}$ group of $\mathrm{EG}$ and the $-\mathrm{SO}_{3}$ group of the anion. This configuration may increase the fluidity of the mixture but not necessarily the ionic dissociation. Moreover, diffusion NMR results indicate the occurrence of local proton dynamics, which arise from a proton exchange between the $-\mathrm{NH}$ of the cation and the $-\mathrm{OH}$ of EG, providing the requisite for a long-range Grotthuss mechanism of proton transport.
\end{abstract}

\section{Introduction}

A current challenge in the design of materials for fuel cell applications is finding an electrolyte that is chemically and thermally stable, while sustaining proton conduction in a wide temperature range extending to well above $100{ }^{\circ} \mathrm{C}$, the limit of aqueous electrolytes. In the proton exchange membrane fuel cell (PEMFC) proton conductivity is typically provided by an acidic aqueous electrolyte retained in a nanoporous polymer membrane (e.g. Nafion). Despite performing very well at low temperatures and high hydration levels, this material concept shows a drastic conductivity drop at above $80{ }^{\circ} \mathrm{C}$ due to dehydration. ${ }^{1}$ It is thus desirable to develop materials with a better thermal stability since at higher temperatures the tolerance of the fuel (i.e. the $\mathrm{H}_{2}$ gas) to $\mathrm{CO}$ poisoning is higher and the electrochemical reactions are faster. Protic ionic liquids (PILs) are attracting considerable attention as alternative electrolytes, mainly due to their thermal and electrochemical

Department of Chemistry and Chemical Engineering, Chalmers University of Technology, SE-412 96 Gothenburg, Sweden. E-mail: anna.martinelli@chalmers.se; Tel: +46 (0)317723002

$\dagger$ Electronic supplementary information (ESI) available: Additional data on the phase behavior of the liquid mixtures (DSC), the thermal stability of one liquid mixture (TGA) and a comparison of the effect on ionic conductivity when adding water or EG. Additional diffusional data and physicochemical properties are also provided in table format. See DOI: 10.1039/c8cp03093f stability, low volatility and high concentration of ionic species. By means of their protic site, typically an $\mathrm{NH}$ group on the cation, PILs also form extended H-bonds, which are crucial for an efficient proton conduction mechanism. ${ }^{2}$ Nevertheless, whether or not PILs can provide a long-range proton transport mechanism decoupled from molecular diffusion is an issue of current debate. ${ }^{3,4}$ Along with studies focused on the behaviour of neat PILs, there is now an increasing interest in investigating the effect of adding a second compound. In this context, PIL/water binary mixtures have been studied to measure changes in thermodynamic properties such as phase transition temperatures, density, and viscosity. ${ }^{5,6}$ Some efforts have also been spent to understand how added water affects the ionic conductivity or the self-diffusion of the ions in the PIL, ${ }^{7,8}$ but considerably fewer studies have been focused on explaining these effects in terms of intermolecular interactions. ${ }^{9}$ In some recent works we have shown that the extent by which ionic mobility and ionicity in imidazolium based PILs such as $\mathrm{C}_{2} \mathrm{HImTFSI}$ and $\mathrm{C}_{2} \mathrm{HImTfO}$ are enhanced by added water very much depends on the way water coordinates to the ions. ${ }^{8,9}$ A sparingly explored approach is the addition to imidazolium based PILs of imidazole, $\$$ which compared to water is more basic and has a lower volatility. We have shown that due to its amphoteric character imidazole forms directional H-bonds

† A well known proton conductor, though only at temperatures above $90{ }^{\circ} \mathrm{C}$. 
within the ionic network of the PIL $\mathrm{C}_{2}$ HImTFSI, thus promoting a Grotthuss mechanism of proton transfer decoupled from molecular diffusion. ${ }^{10}$

Another compound that may have beneficial effects on the transport properties of PILs is ethylene glycol, otherwise used as a co-solvent to ILs in various chemical processes to enhance heat transfer properties. ${ }^{11}$ In some studies focused on aprotic ILs based on 1-alkyl-3-methylimidazolium cations it has been shown that the hydroxyl group of ethylene glycol can form hydrogen bonds of different strength with the aromatic protons of the cation. ${ }^{12}$ Another study indicates that the proton at $\mathrm{C}^{2}$ position on the imidazolium ring, as well as the type of associated anion, play a key role in the strength of the hydrogen bond established between ethylene glycol and the imidazolium cation. ${ }^{13}$ Based on these previous results, the question arises whether ethylene glycol would interact differently with PILs, given their ability to form hydrogen bonds through the more acidic - NH site. To the best of our knowledge, this kind of binary mixtures has scarcely been studied before. Moreover, in view of fuel cell applications an interesting property of ethylene glycol is its lower volatility, hence its higher thermal stability, than water.

In the present paper, we study the effect of adding ethylene glycol to the PIL $\mathrm{C}_{2} \mathrm{HImTfO}$, investigating the entire compositional range. By combining conductivity measurements with pulsed field gradient nuclear magnetic resonance (PFG NMR) spectroscopy, and ${ }^{1} \mathrm{H}$ NMR with vibrational spectroscopy we are able to correlate the observed transport properties with the nature of intermolecular interactions established, with particular attention to the proton exchange mechanism involving the $-\mathrm{NH}$ site.

\section{Experimental}

\subsection{Material}

The protic ionic liquid (PIL) ethylimidazolium triflate $\left(\mathrm{C}_{2}\right.$ HImTfO, $\left.M_{\mathrm{w}}=246 \mathrm{~g} \mathrm{~mol}^{-1}\right)$ was purchased from Io.Li.Tec Germany. Karl Fischer analyses revealed that the content of water in the as purchased sample was $111 \mathrm{ppm}$ (which corresponds to 0.001 water molecules per cation-anion pair). Ethylene glycol (EG, $M_{\mathrm{w}}=62 \mathrm{~g} \mathrm{~mol}^{-1}$ ) was purchased from Sigma Aldrich and used as received. Different EG/ $\mathrm{C}_{2} \mathrm{HImTfO}$ mixtures were prepared with the mole fraction of EG, $\chi$, ranging from 0 to 1 . By eye inspection, all solutions looked clear and homogeneously mixed. The water content of all mixtures was determined by Karl Fischer titration, and was found to be in the mole fraction range $0.002<\chi<0.008$. The molecular structure and atom labelling of $\mathrm{C}_{2} \mathrm{HImTfO}$ and ethylene glycol are shown in Fig. 1.

\subsection{Diffusion NMR}

All the NMR experiments were done on samples contained in standard $5 \mathrm{~mm}$ NMR tubes using a Bruker Avance 600 spectrometer at a magnetic field of $14.1 \mathrm{~T}$. The magnetic field was shimmed using Topspin's topshim routine and fine-tuned

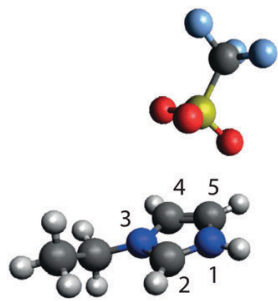

Ethylimidazolium triflate

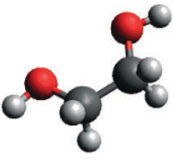

Ethylene glycol
Fig. 1 Chemical structure of ethylimidazolium triflate $\left(\mathrm{C}_{2} \mathrm{HImTfO}\right)$ (left) and ethylene glycol (EG) (right). The atom labelling is also shown. Carbon atoms are shown in grey, oxygen atoms in red, hydrogen atoms in white, nitrogen atoms in blue, sulfur atoms in yellow and fluorine atoms in light blue.

manually. The spectrometer was equipped with ${ }^{1} \mathrm{H}$ and ${ }^{19} \mathrm{~F}$ diffusion probes with a maximum gradient field of $1200 \mathrm{G} \mathrm{cm}^{-1}$. A Pulse Field Gradient Double-Stimulated Echo (diffDSte) pulse sequence with Longitudinal Eddy current Delay (LED) and spoiler gradient pulses was used to reduce convection effects when determining the self-diffusion coefficient. An external chemical shift reference was used and pulse calibration was performed. All diffusion measurements were done at $30{ }^{\circ} \mathrm{C}$. The self-diffusion coefficients were obtained by fitting the decay of the echo signal with the Stejskal-Tanner equation ${ }^{14}$

$$
I=I_{0} \mathrm{e}^{-(\gamma \delta G)^{2} \cdot D \cdot(\Delta-\delta / 3)}
$$

where $I$ is the signal intensity, $I_{0}$ is the signal intensity of spin-echo at zero gradient, $G$ is the gradient strength, $D$ is the self-diffusion coefficient, $\delta$ the length of the gradient pulse, and $\Delta$ is the diffusion time. The maximum gradient used for each sample was set in the range $200-400 \mathrm{G} \mathrm{cm}^{-1}$ depending on the signal attenuation such that 3 to 5 percent of $I_{0}$ remains. The diffusion time $\Delta$ and the pulse duration $\delta$ were set to 100 and $1 \mathrm{~ms}$ for all samples, respectively. 16 transients were averaged with a recycle delay of $5 \mathrm{~s}$ according to the longest specific $T_{1}$ values measured for the different spins at $30{ }^{\circ} \mathrm{C} . T_{2}$ measurements were also done to ensure the time delays are suitable for the pulse sequence used.

\subsection{Conductivity measurements}

Conductivity measurements were performed on a CDM 210 conductivity meter instrument. Consistently with the procedure used for diffusion measurements, the samples were equilibrated at $30{ }^{\circ} \mathrm{C}$ before collecting the data. The instrument was calibrated with an aqueous solution of $\mathrm{KCl}$ at a concentration of $0.01 \mathrm{M}$, while the temperature sensor had a precision of $0.1{ }^{\circ} \mathrm{C}$.

\subsection{Vibrational spectroscopy}

Infrared spectra were collected with a Perkin Elmer spectrometer using the attenuated total reflection (ATR) mode and pouring the solutions over a single-point reflection diamond crystal. For each sample 20 scans were averaged achieving a nominal spectral resolution of $2 \mathrm{~cm}^{-1}$. Where a peak fitting 
procedure was applied, a linear background and a combination of Gaussian functions was used.

Raman spectra were recorded from 100 to $4000 \mathrm{~cm}^{-1}$ using an InVia Reflex Renishaw spectrometer. The $785 \mathrm{~nm}$ laser was used as the excitation source and spectra were obtained from accumulating 4 scans with 10 seconds of exposure each. A peak-fit procedure based on Gaussian type function was employed to extract the position of relevant vibrational modes.

\subsection{Viscosity and density measurements}

The density of the mixtures was measured by an Anton Paar density meter at $30{ }^{\circ} \mathrm{C}$. The viscosity was also measured by an Anton Paar rheometer model MCR 300 with cone and plate geometry of $\phi=50 \mathrm{~mm}$, angle $=1^{\circ}$, and gap $52 \mu \mathrm{m}$ at $30^{\circ} \mathrm{C}$. The shear frequency were adjusted to $10 \mathrm{~Hz}$ for the investigated binary system.

\subsection{Thermogravimetric analysis (TGA)}

The thermal stability of the EG/ $\mathrm{C}_{2} \mathrm{HImTfO}$ mixtures was examined using a Mettler Toledo thermogravimetric analyzer. Standard $70 \mu \mathrm{l}$ alumina crucibles were used for heating the samples at a rate of $10{ }^{\circ} \mathrm{C} \mathrm{min}{ }^{-1}$, covering the temperature range $25-500{ }^{\circ} \mathrm{C}$. Nitrogen was used as the purging gas.

\subsection{Differential scanning calorimetry (DSC)}

Differential scanning calorimetric (DSC) experiments were performed on a Mettler Toledo calorimeter covering the temperature range from $-120{ }^{\circ} \mathrm{C}$ to $+130{ }^{\circ} \mathrm{C}$ and keeping the samples in hermetically sealed aluminum pans. The samples were first quickly cooled from $25{ }^{\circ} \mathrm{C}$ to $-120{ }^{\circ} \mathrm{C}$ at a rate of $-20{ }^{\circ} \mathrm{C} \min ^{-1}$, then DSC traces were recorded during the subsequent heating scan at a rate of $10{ }^{\circ} \mathrm{C} \min ^{-1}$ up to $130{ }^{\circ} \mathrm{C}$. Only the data from the heating scan are presented and discussed in this paper (see the $\mathrm{ESI} \dagger$ file).

\section{Results and discussion}

\subsection{Ionic mobility and ionicity}

Fig. 2 shows the dependence of ionic conductivity on composition in $\mathrm{C}_{2} \mathrm{HImTfO} / \mathrm{EG}$ mixtures, for mole fractions $(\chi)$ of ethylene glycol (EG)

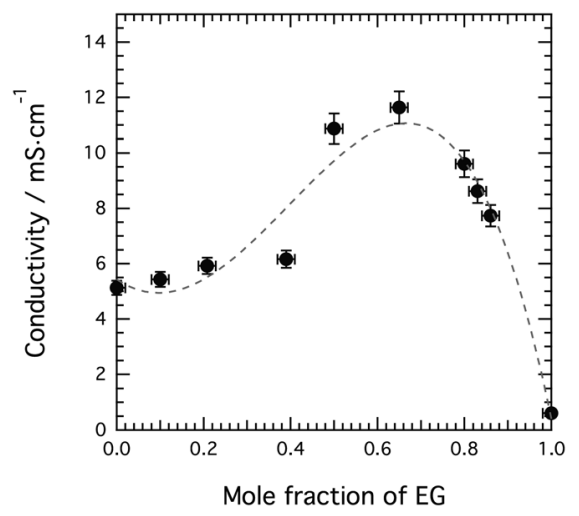

Fig. 2 Dependence of the ionic conductivity on composition in the binary mixtures based on $\mathrm{C}_{2} \mathrm{HImTfO}$ and ethylene glycol. The grey dashed line is simply a guide to the eye. between 0 and 1 , measured at $30{ }^{\circ} \mathrm{C}$. All samples are liquid at this temperature, see Fig. S1 (ESI $\dagger$ ). The addition of EG to the PIL has a moderate effect on conductivity, which displays a maximum at $\chi \approx 0.66$, at which composition the increase is twofold. This nonmonotonic behaviour is rationalised by a competition between dissociation, concentration and mobility of the charge carriers, and has previously been observed for several imidazolium based ionic liquids upon addition of water or ethanol. ${ }^{7}$ However, the extent by which the conductivity of $\mathrm{C}_{2} \mathrm{HImTfO}$ is increased by EG is markedly lower than observed by adding water, ${ }^{8}$ which results in a more than tenfold increase with a maximum at $\chi \approx 0.9$ (see Fig. S2, $\mathrm{ESI} \dagger)$. This difference most likely arises from the higher viscosity of EG as compared to water, $\S$ which limits the overall mobility of the ions.

The ionic mobility, i.e. the self-diffusion coefficient, $D$, of all ionic species could be measured by PFG-NMR spectroscopic experiments. The self-diffusion coefficients of ethylene glycol, $D_{\mathrm{EG}}$, and the cation, $D_{\text {cation, }}$ can be measured from their separate ${ }^{1} \mathrm{H}$ NMR signals, Fig. 3A. The reported values of $D_{\text {cation }}$ are an average obtained from the NMR resonances of aromatic and aliphatic protons, excluding the resonance of the $-\mathrm{NH}$ group that may have a different behaviour $\left(D_{\mathrm{NH}}\right.$, vide infra). The same procedure could be applied for EG, but due to some overlap between the resonance of its $\mathrm{OH}$ groups with those of the ionic liquid for some $\chi$ values, only diffusion values extracted from the resonance of the $\mathrm{CH}_{2}$ protons are presented and discussed. $\uparrow$ Self-diffusion values are shown in Fig. 4A and reveal that the addition of EG increases the diffusivity of all molecular species, as a direct consequence of a decreased viscosity (see Table S1 in the ESI $\dagger$ file). The self-diffusion of

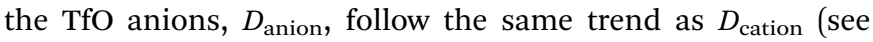
experimental values in Table S2 of the ESI $\dagger$ file) but are always slightly lower, as typically found in imidazolium based ionic liquids with anions and cations of comparable size. We also note that EG diffuses faster than the cation at all compositions, i.e. that $D_{\mathrm{EG}}>D_{\text {cation, }}$ which is consistent with the smaller size of EG. Moreover, the self-diffusion values extracted from the -NH resonance, $D_{\mathrm{NH}}$, are comparable within the experimental errors to those of $D_{\text {cation }}$ at low concentrations of EG, but show a deviation for values of $\chi$ greater than 0.4 . This effect is better visualised in Fig. $4 \mathrm{~B}$, where the $D_{\mathrm{NH}} / D_{\text {cation }}$ ratios are plotted as a function of composition. It should be noted that a $D_{\mathrm{NH}} / D_{\text {cation }}$ ratio significantly higher than one indicates a proton motion decoupled from molecular diffusion and is a prerequisite for the Grotthuss mechanism of proton transfer. Hence, Fig. 4B shows that this may be the case for binary mixtures where $\chi$ is greater than 0.4. The Grotthuss mechanism of proton transport, not observed in the pure PIL, may be induced by the formation of new hydrogen bonds that involve the $-\mathrm{NH}$ group of the cation. Interestingly, the FWHM (full width at half maximum) of the $\mathrm{NH}$ resonance in the ${ }^{1} \mathrm{H}$ NMR spectrum

$\S$ At $25{ }^{\circ} \mathrm{C}$ the viscosity of EG is $16 \mathrm{mPa}$, while that of water is $0.9 \mathrm{mPa}$. T Nevertheless, a careful analysis of the NMR data reveals equal values, within experimental errors, for the self-diffusion extracted from the $\mathrm{OH}$ and the $\mathrm{CH}_{2}$ resonances of EG. 

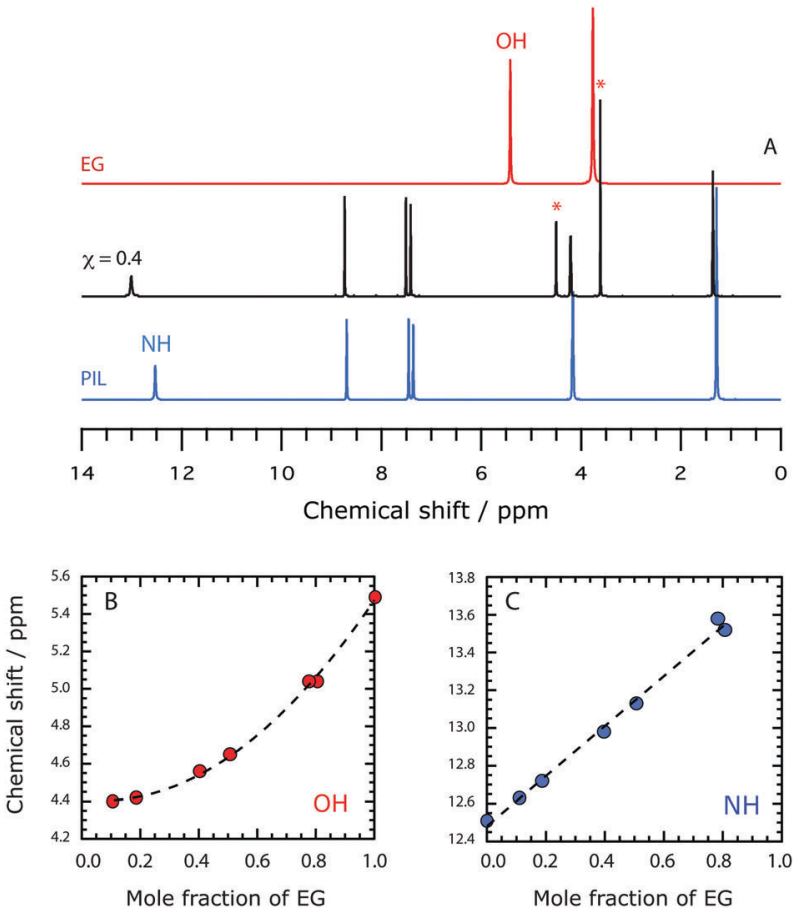

Fig. 3 (A) Selected ${ }^{1} H$ NMR spectra, i.e. those collected for pure EG, the $\mathrm{EG} / \mathrm{C}_{2} \mathrm{HImTfO}$ mixture with $\chi=0.4$ and pure $\mathrm{C}_{2} \mathrm{HImTfO}$. The chemical shifts were referenced to tetramethylsilane (TMS) and assigned as follows: $\mathrm{NH}$ at $12-14 \mathrm{ppm} ; \mathrm{C}^{2} \mathrm{H}$ at $8.6 \mathrm{ppm} ; \mathrm{C}^{4,5} \mathrm{H}$ at 7-7.5 ppm; $\mathrm{CH}_{2}$ at $4.2 \mathrm{ppm}$; $\mathrm{CH}_{3}$ at $1.3 \mathrm{ppm}$; $\mathrm{OH}$ of $\mathrm{EG}$ at $5.5 \mathrm{ppm}$ and $\mathrm{CH}_{2}$ of $\mathrm{EG}$ at $3.5 \mathrm{ppm}$. The stars in the intermediate spectrum (i.e. that of the mixture with $\chi=0.4$ ) indicate resonance peaks arising from EG. (B) ${ }^{1} \mathrm{H} N M R$ chemical shift of the $\mathrm{OH}$ proton as a function of added ethylene glycol (EG). (C) ${ }^{1} \mathrm{H}$ NMR chemical shift of the $\mathrm{NH}$ proton as a function of added ethylene glycol (EG).

shows a compositional dependence, and increases with the amount of EG, Fig. 4C. The results show a sharp increase at around $\chi>0.4$, suggesting that effective proton exchange between the cation and EG does not happen at lower EG mole fractions, presumably because of its preferred interaction with the anion. It is worth mentioning that even for the broadest peaks corresponding to the highest exchange rates, we are still in the slow exchange regime compared to the NMR time scale. The proton exchange events between the $\mathrm{NH}$ group of the cation and the $\mathrm{OH}$ of EG with $8.6 \mathrm{ppm}$ separation in a $500 \mathrm{MHz}$ spectrometer is calculated to occur in a time scale longer than $0.23 \mathrm{~ms}$. $\|$

Also the ionicity, an indicator of the ionic dissociation in ILs, shows an interesting composition dependence. This has been calculated using the two very common methods of (i) comparing the experimentally measured molar conductivity, $\Lambda_{\text {imp }}$, to the behaviour of an ideal aqueous solution of $\mathrm{KCl}$ in a Walden plot and (ii) by calculating the $\Lambda_{\mathrm{imp}} / \Lambda_{\mathrm{NMR}}$ ratio.**

$\|$ This exchange time is calculated as $1 /\left(\delta_{\mathrm{NH}}-\delta_{\mathrm{OH}}\right) \cdot 500 \mathrm{MHz}$, where $\delta_{\mathrm{NH}}$ is the chemical shift of the $\mathrm{NH}$ group in ethylimidazolium, $\delta_{\mathrm{OH}}$ is the chemical shift of the $\mathrm{OH}$ group in ethylene glycol, and $500 \mathrm{MHz}$ is the spectrometer's frequency.

** $\Lambda_{\mathrm{NMR}}$ is estimated from the measured diffusion values using the Nernst-Einstein relation $\Lambda_{\mathrm{NMR}}=F^{2} / R T \cdot\left(D_{\text {cation }}+D_{\text {anion }}\right)$. In an electrolyte with entirely dissociated ions, the $\Lambda_{\mathrm{imp}} / \Lambda_{\mathrm{NMR}}$ ratio is expected to be equal to unity, whilst in ionic liquids values lower than one are commonly observed due to partial ionic association.
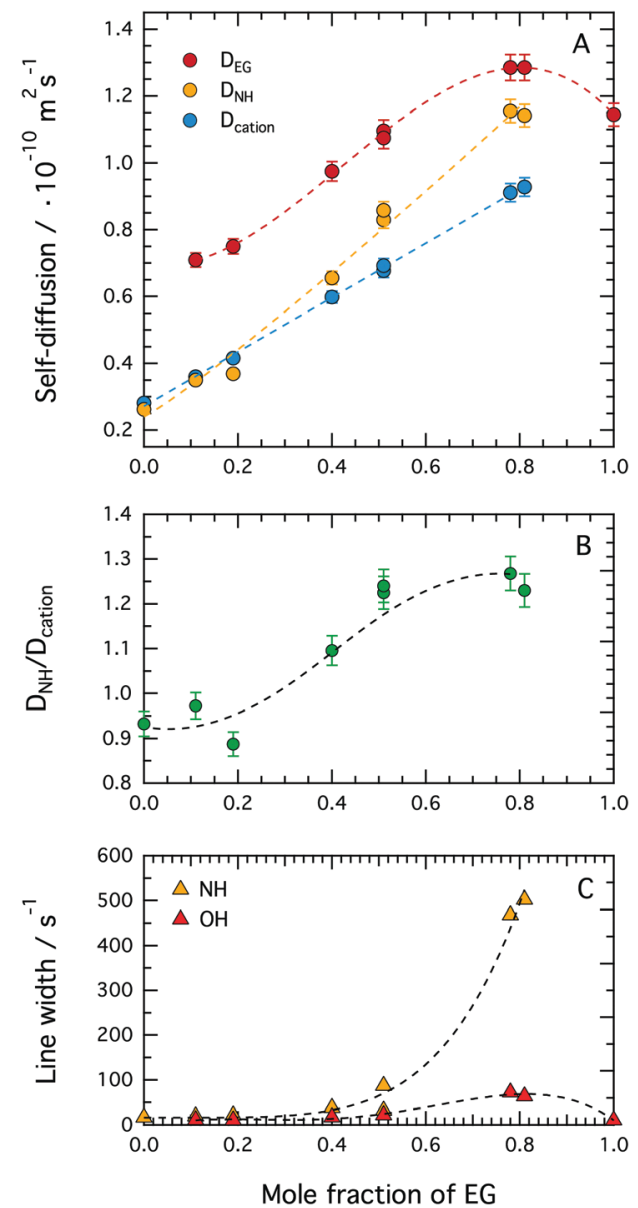

Fig. 4 (A) Self-diffusion coefficients measured at $30{ }^{\circ} \mathrm{C}$ for the $\mathrm{C}_{2} \mathrm{HIm}$ cation $\left(D_{\text {cation }}\right)$, the exchangeable $\mathrm{NH}$ proton $\left(D_{\mathrm{NH}}\right)$ and ethylene glycol $\left(D_{\mathrm{EG}}\right)$ as a function of added EG. (B) Dependence of the $D_{\mathrm{NH}} / D_{\text {cation }}$ ratio on the amount of added EG. (C) Dependence of the line width for the resonances of the $-\mathrm{NH}$ and $-\mathrm{OH}$ protons on the amount of added $\mathrm{EG}$. Line width values are also given in a tabulated form in Table S2 (ESI $)$. Dashed lines are guide to the eye only.

The results from these two approaches, based on entirely independent measurements, are shown in Fig. 5 (see also Table $\mathrm{S} 2$ in the ESI $\dagger$ file for tabulated values). It is striking that both methods show first a decrease of ionicity, for $\chi<0.4$, followed by a discontinuous increase of ionicity for $\chi>0.4$. Changes of ionicity in PILs due to the addition of EG have previously been investigated only by Thawarkar et al., ${ }^{15}$ who also reported ionicity values lower than one, spanning between 0.33 and 0.64 depending on the anion associated to the 1-methylimidazolium cation. The authors also noted a decrease in ionicity with temperature in the investigated EG/PIL mixtures, which is a typical behaviour also for neat PILs. The increase in ionicity that we report here is an effect of composition and reflects the complexity of simultaneous ion-ion and ion-EG interactions.

\subsection{Intermolecular interactions and $\mathrm{H}$-bonds}

A plausible site of interaction for the $-\mathrm{NH}$ group of the cation, which acts as a proton donor, is the $-\mathrm{OH}$ group of EG, which can act both as proton donor and proton acceptor. In fact, in its 

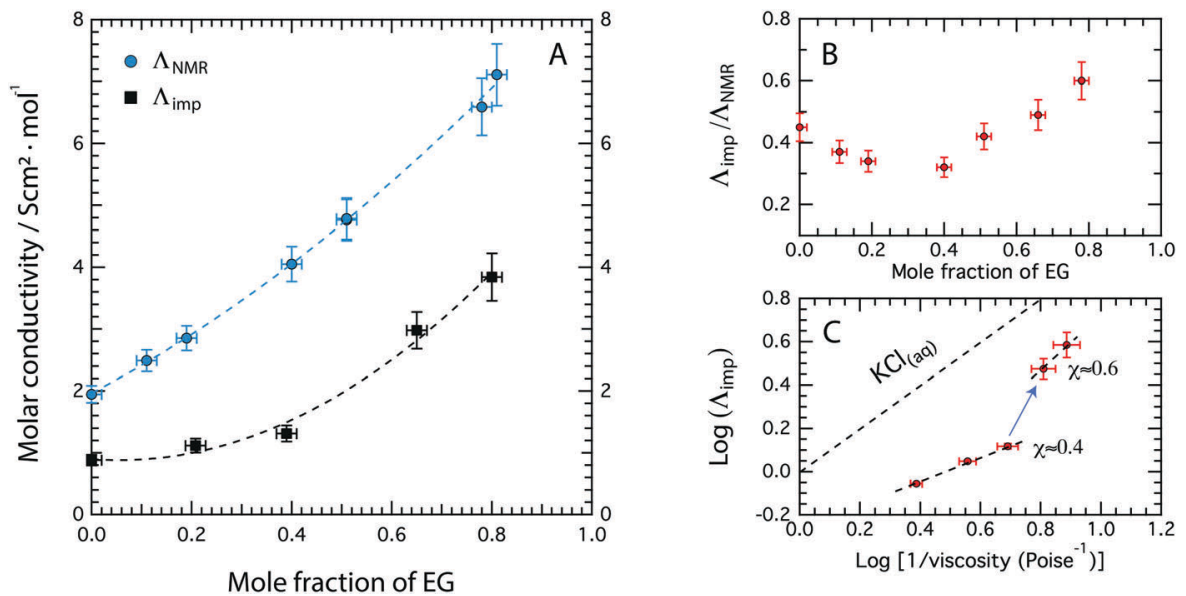

Fig. 5 (A) Molar conductivities, $\Lambda_{\mathrm{m}}$, calculated from experimental conductivity values $\left(\Lambda_{\mathrm{imp}}\right)$ and deduced from self-diffusion values $\left(\Lambda_{\mathrm{NMR}}\right)$. (B) lonicity values calculated as the $\Lambda_{\mathrm{imp}} / \Lambda_{\mathrm{NMR}}$ ratio. (C) lonicity values obtained from $\Lambda_{\mathrm{imp}}$ and shown in a Walden plot.

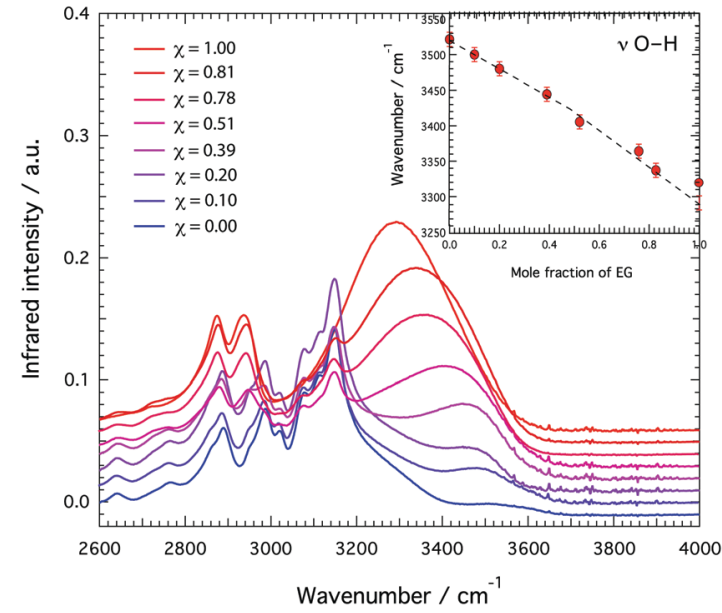

Fig. 6 High frequency range of the infrared spectra for the mixtures of ethylene glycol and $\mathrm{C}_{2} \mathrm{HImTfO}$ with the EG mole fraction, $\chi$, varying from 0 to 1.

pure state EG is known to form strong intramolecular hydrogen bonds that involve the terminal $\mathrm{O}$ and $\mathrm{H}$ atoms. ${ }^{16,17}$ Infrared spectroscopy shows a red shift for the $\mathrm{O}-\mathrm{H}$ stretching mode (observed in the frequency range $3200-3600 \mathrm{~cm}^{-1}$ ) with increasing EG content, Fig. 6, which is nicely confirmed by the shift to higher frequencies of the $\mathrm{OH}$ proton resonance observed in the ${ }^{1} \mathrm{H}$ NMR spectrum, Fig. 3B. The NH proton resonance shows a shift to higher frequencies as well, Fig. 3C. $\dagger \dagger$ The fate of the TfO anion is most easily investigated in the Raman spectrum, where the symmetric S-O stretching modes $\left(\nu_{\mathrm{S}} \mathrm{SO}_{3}\right)$ appear as a strong feature at $\sim 1033 \mathrm{~cm}^{-1}$, Fig. 7 . This mode is sensitive to local interactions ${ }^{18}$ and has therefore been chosen to understand the effect of adding EG. Due to an interference in this frequency range from a vibrational mode of the cation, ${ }^{9}$ a peak fitting procedure was employed based on a linear background

$\dagger \dagger$ By contrast, the strong overlap in the infrared spectra of $\mathrm{N}-\mathrm{H}, \mathrm{C}^{\text {ring }}-\mathrm{H}$ and $\mathrm{O}-\mathrm{H}$ stretching modes does not allow a reliable analysis of the $\nu \mathrm{N}-\mathrm{H}$ frequency. and two Gaussian functions, centred at $1029 \mathrm{~cm}^{-1}$ (contribution from the cation) and at $1033 \mathrm{~cm}^{-1}$ (the $\mathrm{S}-\mathrm{O}$ stretch of the TfO anion). This analysis reveals that upon addition of EG the $\mathrm{S}-\mathrm{O}$ stretching mode $\left(\nu_{\mathrm{S}} \mathrm{S}-\mathrm{O}_{3}\right)$ shifts to lower wavenumbers, reflecting an elongation of the $\mathrm{S}-\mathrm{O}$ bonds and thus $\mathrm{TfO} \cdots \mathrm{C}_{2} \mathrm{HIm}$ interactions altered by the presence of EG.

To summarise, Raman, infrared and NMR spectroscopy reveal changes in the nature of hydrogen bonds established in the binary mixture, with the intramolecular hydrogen bonds typical of pure EG weakened by the presence of the PIL ${ }^{16}$ (see blue shift with increasing PIL content in Fig. 6), as well as more acidic $\mathrm{N}-\mathrm{H}$ groups (see trends in Fig. $3 \mathrm{C}$ ) and elongated $\mathrm{S}-\mathrm{O}$ bonds (see inset of Fig. 7) induced by the presence of EG. A simplified scheme representing an hydrogen bond configuration able to explain the observed spectroscopic features is given in Fig. 8. This scheme also aims to illustrate that for $\chi>0.4$, i.e. for amounts of EG exceeding a $1: 1$ (EG : PIL) ratio, EG molecules tend to interact with each other re-establishing stronger intra- and inter-molecular hydrogen bonds. This agrees well with the trend change observed at $\chi>0.4$ for the chemical shift of the $\mathrm{OH}$ protons in EG (Fig. 3B), a trend change also partially observed in infrared for the $\mathrm{O}-\mathrm{H}$ stretching mode (inset of Fig. 6). That EG interferes with the native cation-anion interactions was recently discussed by Thawarkar et $a .^{15}$ as a function of temperature and for one specific EG/PIL composition, although for different cation-anion pairs than the one investigated here. In the present study we focus on the composition dependence of local interactions, and show that these are able to also affect the local dynamics, and thus the ionicity. Our results evidence two compositional ranges. For $\chi$ values below 0.4 , fluidity and diffusivity increase, while ionicity decreases as a result of ionic association, likely induced by EG molecules holding together both cations and anions through shared hydrogen bonds, see Fig. 8. In this range EG molecules are assumed to distribute homogeneously within the ionic network. For $\chi$ values above 0.4, EG molecules start to also interact with each other since the interions sites of interaction are saturated. This results in $\mathrm{OH}$ groups more strongly hydrogen bonded, but also in faster local dynamics 


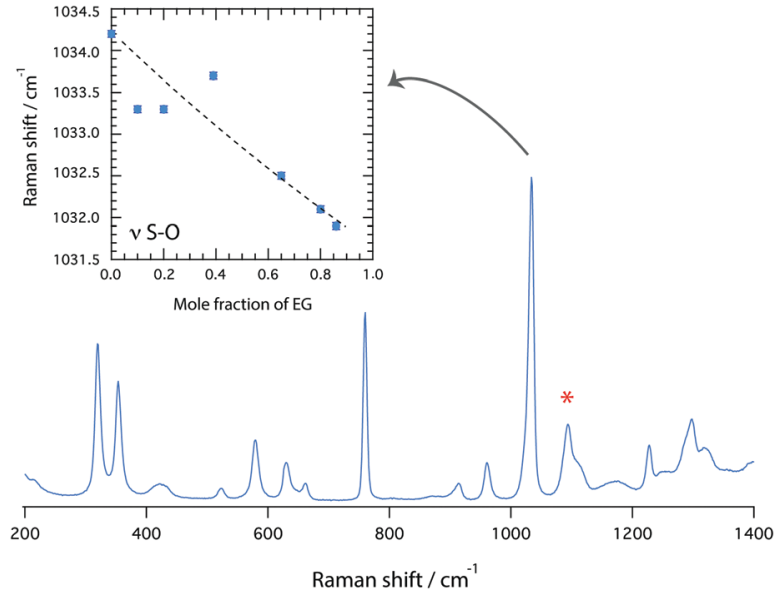

Fig. 7 A Raman spectrum collected for the binary mixture $E G / C_{2} \mathrm{HImTfO}$ with $\chi=0.51$ showing the spectral region where the symmetric $\mathrm{S}-\mathrm{O}$ stretching modes are found, i.e. at $\sim 1034 \mathrm{~cm}^{-1}$. The asterisk indicates a contribution from EG.
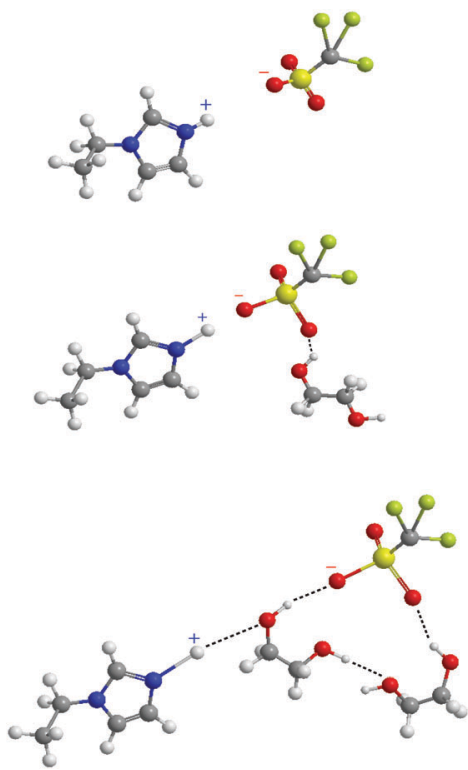

Fig. 8 Tentative illustration of the possible $\mathrm{H}$-bonds formed due to ion-ion, ion-EG and EG-EG interactions at different representative compositions.

involving protons. This is manifested by a line broadening of the $(\mathrm{N}) \mathrm{H}$ resonance in the ${ }^{1} \mathrm{H}$ NMR spectrum and an increased $D_{\mathrm{NH}} / D_{\text {cation }}$ ratio (Fig. $4 \mathrm{~B}$ and C). In other words, the hydrogen bond configuration established at concentrations of excess EG seems to favour local proton dynamics decoupled from molecular diffusion. The occurrence of these proton transfer events is also at the origin of the ionicity increase observed when $\chi$ is varied from 0.4 to 0.6 (Fig. 5C). Notably, this is different from the effect of water that when added to $\mathrm{C}_{2} \mathrm{HImTfO}$ results in a marginal ionicity change. ${ }^{8}$

\section{Conclusions}

The effect of adding ethylene glycol (EG) on the transport properties of the protic ionic liquid (PIL) ethylimidazolium triflate, $\mathrm{C}_{2} \mathrm{HImTfO}$, has been investigated. Ethylene glycol is a liquid prone to establishing intra- and intermolecular $\mathrm{H}$-bonds with a good thermal stability (boiling point at $197^{\circ} \mathrm{C}$ ) and was therefore chosen as a second component to form a binary mixture with potential use as a proton conducting material. EG- $\mathrm{C}_{2}$ HImTfO interactions are established mainly through the $\mathrm{NH}$ site, in contrast to aprotic ionic liquids which interact mainly through the aromatic $\mathrm{C}^{2,4,5}-\mathrm{H}$ sites, an insight that is provided by ${ }^{1} \mathrm{H}$ NMR chemical shifts analysis. The nature of hydrogen bonding is such to decrease ionicity at low EG contents, despite an increase in ionic mobility. At EG contents higher than equimolar, however, ionicity increases as a consequence of faster local proton dynamics, evidenced by excess diffusion values (i.e. $D_{\mathrm{NH}}>D_{\text {cation }}$ ) and line widths $\left(\mathrm{FWHM}_{\mathrm{NH}}\right.$ $>$ FWHM $_{\text {ringH }}$ ). Whether or not a longer range proton transport based on the Grotthuss mechanism also contributes to the measured $D_{\mathrm{NH}}$ value, remains to be verified by more advanced techniques such as quasi elastic neutron scattering, that together with diffusion NMR allows covering a larger time scale range. With respect to applications in fuel cells, the binary mixture with the highest ionic conductivity $(\chi=0.6)$ is thermally stable up to $\sim 110{ }^{\circ} \mathrm{C}$ (Fig. S3, ESI $\dagger$ ), a modest improvement with respect to aqueous based electrolytes that in polymer membranes are limited to below $80{ }^{\circ} \mathrm{C}$.

\section{Conflicts of interest}

There are no conflicts to declare.

\section{Acknowledgements}

Funding from the Chalmers Area of Advance Energy, the Knut \& Alice Wallenberg Foundation (2016-0220) and Formas (Leaders of the Future Award, grant no. 2016-01189) is kindly acknowledged. The authors also kindly thank professor Istvan Furo for providing machine time with the NMR spectrometer.

\section{References}

1 J. R. O'Dea, N. J. Economou and S. K. Buratto, Macromolecules, 2013, 46, 2267-2274.

2 W. Münch, K. D. Kreuer, W. Silvestri, J. Maier and G. Seifert, Solid State Ionics, 2001, 145, 437-443.

3 T. Burankova, R. Hempelmann, V. Fossog, J. Ollivier, T. Seydel and J. P. Embs, J. Phys. Chem. B, 2015, 119, 10643-10651.

4 M. L. Hoarfrost, M. Tyagi, R. A. Segalman and J. A. Reimer, J. Phys. Chem. B, 2012, 116, 8201-8209.

5 M. Anouti, J. Jacquemin and D. Lemordant, Fluid Phase Equilib., 2010, 297, 13-22.

6 M. Anouti, M. Caillon-Caravanier, Y. Dridi, C. Hardacre and D. Lemordant, J. Chem. Thermodyn., 2009, 41, 799-808.

7 E. Rilo, J. Vila, S. Garcia-Garabal, L. M. Varela and O. Cabeza, J. Phys. Chem. B, 2013, 117, 1411-1418. 
8 N. Yaghini, L. Nordstierna and A. Martinelli, Phys. Chem. Chem. Phys., 2014, 16, 9266-9275.

9 N. Yaghini, J. Pitawala, A. Matic and A. Martinelli, J. Phys. Chem. B, 2015, 119, 1611-1622.

10 N. Yaghini, V. Gomez-Gonzalez, L. M. Varela and A. Martinelli, Phys. Chem. Chem. Phys., 2016, 18, 23195-23206.

11 A. P. C. Ribeiro, S. I. C. Vieira, J. M. França, C. S. Queirós, E. Langa, M. J. V. Lourenço, S. M. S. Murshed and C. A. Nieto de Castro, Thermal properties of Ionic liquids and Ionanofluids, InTech, 2011, pp. 37-60.

12 B. Kumar, T. Singh, K. S. Rao, A. Pal and A. Kumar, J. Chem. Thermodyn., 2012, 44, 121-127.
13 A. Pal, B. Kumar and T. Singh Kang, Fluid Phase Equilib., 2013, 358, 241-249.

14 E. O. Stejskal and J. E. Tanner, J. Chem. Phys., 1965, 42, 288-292.

15 S. Thawarkar, N. Khupse and A. Kumar, ChemPhysChem, 2016, 17, 1006-1017.

16 T. Singh, K. S. Rao and A. Kumar, ChemPhysChem, 2011, 12, 836-845.

17 V. Crupi, M. P. Jannelli, S. Magazu, P. Migliardo and D. Sirna, Mol. Phys., 1995, 84, 645-652.

18 C. M. Burba, N. M. Rocher and R. Frech, J. Phys. Chem. B, 2009, 113, 11453-11458. 